\title{
Spontaneous Action Representation in Smokers when Watching Movie Characters Smoke
}

\author{
Dylan D. Wagner, ${ }^{1}$ Sonya Dal Cin, ${ }^{2}$ James D. Sargent, ${ }^{2}$ William M. Kelley, ${ }^{1}$ and Todd F. Heatherton ${ }^{1}$ \\ ${ }^{1}$ Department of Psychological and Brain Sciences and ${ }^{2}$ Norris Cotton Cancer Center, Dartmouth Hitchcock Medical School, Dartmouth College, Hanover, \\ New Hampshire 03755
}

Do smokers simulate smoking when they see someone else smoke? For regular smokers, smoking is such a highly practiced motor skill that it often occurs automatically, without conscious awareness. Research on the brain basis of action observation has delineated a frontoparietal network that is commonly recruited when people observe, plan, or imitate actions. Here, we investigated whether this action observation network would be preferentially recruited in smokers when viewing complex smoking cues, such as those occurring in motion pictures. Seventeen right-handed smokers and 17 nonsmokers watched a popular movie while undergoing functional magnetic resonance imaging. Using a natural stimulus, such as a movie, allowed us to keep both smoking and nonsmoking participants naive to the goals of the experiment. Brain activity evoked by movie scenes of smoking was contrasted with nonsmoking control scenes that were matched for frequency and duration. Compared with nonsmokers, smokers showed greater activity in left anterior intraparietal sulcus and inferior frontal gyrus, regions involved in the simulation of contralateral hand-based gestures, when viewing smoking versus control scenes. These results demonstrate that smokers spontaneously represent the action of smoking when viewing others smoke, the consequence of which may make it more difficult to abstain from smoking.

\section{Introduction}

For smokers, the action of smoking a cigarette is a highly reinforced and overlearned behavior that has been acquired through thousands of exposures to nicotine. After extensive practice, these actions become automatized, allowing smokers to perform them effortlessly and even without awareness (Field et al., 2006). The automaticity of smoking behavior has important consequences for nicotine dependence and relapse but has largely been overlooked in favor of research on cue-induced craving. This is somewhat surprising in light of evidence that automatic smoking is an important predictor of consumption (Russell et al., 1974) and relapse (Berlin et al., 2003) in smokers attempting to quit. Although cue-induced craving is an important contributor to smoking relapse, exposure to smoking cues may also trigger automatic smoking behaviors (Tiffany, 1990). Simply observing another person smoking might lead smokers to spontaneously mimic the action of smoking.

Psychologists have long theorized that observing actions increases the likelihood that a person will perform those actions

\footnotetext{
Received Oct. 2, 2010; revised Nov. 8, 2010; accepted Nov. 9, 2010.

This research was supported by a grant from the National Institute on Drug Abuse (R01DA22582) to T.F.H., a Cancer Control Research Program Prouty Pilot Grant from the Norris Cotton Cancer Center to S.D.C. and J.D.S., and a Strategic Training Fellowship in Tobacco Research from the Canadian Institutes of Health Research awarded to S.D.C We thank Keilah Worth for suggestions during the design and analysis of this research. D.D.W. collected, analyzed, and interpreted the data and wrote the first draft of the manuscript; D.D.W., S.D.C., W.M.K., and T.F.H. designed the experiment; and S.D.C., J.D.S., and T.F.H. contributed to the interpretation of the data and to the final version of the manuscript.

Correspondence should be addressed to Dylan D. Wagner, Department of Psychological and Brain Sciences, Moore Hall, Dartmouth College, Hanover, NH 03755. E-mail: dylan.d.wagner@dartmouth.edu.

S. Dal Cin's present address: Department of Communication Studies and the Institute for Social Research, University of Michigan, Ann Arbor, MI 48109.

DOI:10.1523/JNEUROSCI.5174-10.2011

Copyright $\odot 2011$ the authors $\quad 0270-6474 / 11 / 310894-05 \$ 15.00 / 0$
}

(James, 1890). Extensive research in experimental psychology has demonstrated that simple environmental cues can elicit behavior without the participant's awareness (Bargh and Ferguson, 2000). For example, seeing someone scratch their faces increases the likelihood that the observer will spontaneously mimic that same action without any awareness that they are doing so (Chartrand and Bargh, 1999).

Neuroimaging research on action observation has shown that observing goal-directed actions recruits the superior parietal and lateral prefrontal cortices. This action observation network $(\mathrm{AON})$ is recruited when participants observe meaningful actions (Hamilton and Grafton, 2006), mentally plan actions (Decety et al., 1994; Moll et al., 2000), imitate the actions of others (Buccino et al., 2004), and when experts (e.g., dancers) observe familiar movements (Calvo-Merino et al., 2005; Cross et al., 2006). Two regions of the AON are specifically involved in representing goal-directed manual actions. The anterior intraparietal sulcus (aIPS) and the lateral inferior frontal gyrus (IFG; BA 45/46) show greater activity when observing (Rizzolatti et al., 1996; Johnson-Frey et al., 2003; Shmuelof and Zohary, 2005) and planning (Grafton et al., 1996; Johnson-Frey et al., 2005) manual actions such as grasping.

Surprisingly, few neuroimaging studies of smoking cue reactivity have reported activity in the AON. Smoking is an inherently manual skill and therefore it would be expected that observing smoking cues would recruit regions involved in the representation of hand-based actions (e.g., the aIPS and IFG). A possible explanation for this lack may be the common use of static cues (e.g., photographs) that often mix images of smoking actions with images of smoking paraphernalia (e.g., ashtrays). Prior research suggests that smoking in movies is an especially potent 


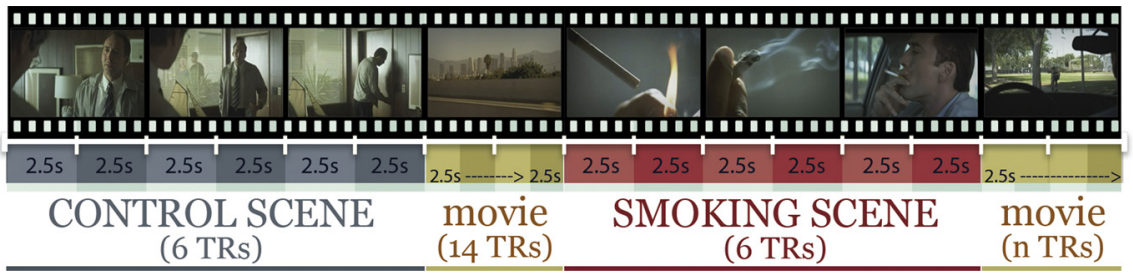

Figure 1. Schematic of the study design. Smoking and nonsmoking control scenes were matched for duration and frequency per run.

smoking cue that increases cigarette craving (Sargent et al., 2009) and consumption (Shmueli et al., 2010). Here, we capitalized on the rich dynamic smoking cues present in popular movies to examine smoking cue-related brain activity in the AON. In line with the research outlined above, we predicted that smokers, compared with nonsmokers, would spontaneously activate the $\mathrm{AON}$ when viewing movie smoking.

\section{Materials and Methods}

Subjects. Twenty right-handed smokers and 21 nonsmokers were recruited to participate in the present study. Participants had normal or corrected-to-normal visual acuity, no history of neurological problems, and were not currently taking medication for blood pressure, depression, or other psychiatric disorders. Three smokers and four nonsmokers were excluded from the analysis due to excessive head motion (four subjects) or scanner artifact (three subjects), leaving a total of 17 smokers (12 women; mean age, 23.1 years) and 17 nonsmokers ( 15 women; mean age, 21.4 years). Nonsmokers were identified on the basis of having never been a regular or casual smoker and having smoked less than a hundred cigarettes in their lifetime. Smokers were daily smokers who smoked on average 10 cigarettes or more a day and were not currently attempting to quit smoking or taking medication to quit smoking (e.g., Zyban). All participants gave informed consent in accordance with the guidelines set by the Committee for the Protection of Human Subjects at Dartmouth College.

Stimuli. Stimuli consisted of the first $30 \mathrm{~min}$ of footage from the 2003 film Matchstick Men. This film was selected from a database of $>420$ top-grossing Hollywood films dating back to 1988 (Dalton et al., 2003). The films in this database have undergone content analysis for scenes of substance use (smoking, alcohol, and drugs), violence, and sexual content (Sargent et al., 2008). Matchstick Men was chosen from this database as a film that contained numerous scenes of smoking but was otherwise low in alcohol use, violence, and sexual content.

Because of scanner run-length limitations, the film was edited into three 10 min segments; each segment was preceded and followed by $60 \mathrm{~s}$ of null event trials consisting of a white fixation cross against a black background. In total, there were $110 \mathrm{~s}$ of on-screen smoking distributed throughout the three segments. Participants listened to the audio soundtrack of the film via a pair of MRI compatible headphones (Resonance Technology).

Procedure. Mass advertising was used to recruit smokers and nonsmokers. All participants participated in a telephone prescreening session. Participants were contacted $\sim 1$ week after the prescreening session and invited to participate in a study on the neural correlates of watching movies. This was done to ensure that participants were unaware of selection criteria (i.e., smoking status) and the nature of the study at the time of scanning. All participants were scanned in the morning and were contacted the night before and given a cover story in which they were informed that it was important for accurate measurement of brain activity that they refrain from drinking caffeinated drinks, smoking tobacco, or consuming alcohol on the morning before scanning, thus ensuring that smokers would arrive craving nicotine. At the time of scanning, participants' only instructions were to remain awake and pay attention to the film. Afterward, participants completed a short debriefing consisting of a 100-point Likert scale of postscan cigarette craving (smokers only) and a questionnaire assessing their impressions about the film and its protagonists.

Image acquisition. Magnetic resonance imaging was conducted with a Philips Achieva 3.0 tesla scanner using an eight-channel phased array coil. Structural images were acquired using a T1-weighted MP-RAGE protocol [160 sagittal slices; repetition time (TR), $9.9 \mathrm{~ms}$; echo time (TE), $4.6 \mathrm{~ms}$; flip angle, $8^{\circ} ; 1 \times 1 \times 1 \mathrm{~mm}$ voxels]. Functional images were acquired using a $2^{*}$-weighted echo-planar sequence (TR, $2500 \mathrm{~ms}$; TE, $35 \mathrm{~ms}$; flip angle, $90^{\circ}$; field of view, $24 \mathrm{~cm})$. For each participant, three runs of 288 whole-brain volumes ( 30 axial slices per whole-brain volume, $4.5 \mathrm{~mm}$ thickness, $0.5 \mathrm{~mm}$ gap; $3 \times 3 \mathrm{~mm}$ in-plane resolution) were collected.

Image preprocessing. $\mathrm{fMRI}$ data were analyzed using the general linear model for event-related designs in SPM8 (Wellcome Department of Cognitive Neurology, London, UK). For each functional run, data were preprocessed to remove sources of noise and artifact. Images were corrected for differences in acquisition time between slices and realigned within and across runs via a rigid body transformation to correct for head movement. Images were then unwarped to reduce residual movement-related image distortions not corrected by realignment. Functional data were normalized into a standard stereotaxic space ( $3 \mathrm{~mm}$ isotropic voxels) based on the SPM8 EPI template that conforms to the ICBM 152 brain template space (Montreal Neurological Institute) and approximates the Talairach and Tournoux atlas space. Finally, normalized images were spatially smoothed ( $6 \mathrm{~mm}$ full-width-at-half-maximum) using a Gaussian kernel to increase the signal-to-noise ratio and to reduce the impact of anatomical variability not corrected for by stereotaxic normalization. Volumes were inspected for scanner and motion-related artifact based on examination of the realignment parameters and voxelwise standard deviations for each run and subject.

Modeling of conditions. Modeling continuous stimuli, such as films, presents a special challenge for the analysis of functional neuroimaging data. Moran and colleagues (2004) successfully used a method for the analysis of brain activity time-locked to specific events (e.g., humor detection and appreciation) in participants viewing full television episodes. In the present study, we used a similar approach by examining brain activity time-locked to smoking and nonsmoking scenes. Seventeen discrete smoking scenes were identified with durations ranging from 2.5 to $30 \mathrm{~s}$ (total time, $117 \mathrm{~s}$ ). An equal duration of nonsmoking scenes were randomly selected from the remaining portion of the film and matched for duration and within-run frequency to the smoking scenes (Fig. 1). The remaining portion of the film was explicitly modeled but was not used for further comparison. As our model was constrained by the specific temporal sequence of smoking scenes in the film, we were unable to explicitly optimize our design for maximum estimability of the conditions of interest. However, the natural temporal order of smoking and control scenes was such that the conditions were inherently jittered (correlation between predictors was 0.09).

Data analysis. For each participant, a general linear model incorporating task effects and covariates of no interest (a session mean, a linear trend to account for low-frequency drift, and six movement parameters derived from realignment correction) was convolved with a canonical hemodynamic response function (HRF) and used to compute contrast images (containing weighted parameter estimates) for the comparison of smoking scenes versus control scenes at each voxel.

Contrast images were entered into a second-level, random-effects analysis with participant (smokers and nonsmokers) treated as the random effect. The resulting whole-brain statistical parametric map representing regions in which activity was greater for smoking versus control scenes for all participants was thresholded at $p<0.005$, uncorrected with an extent threshold of 10 contiguous voxels. This combined map of smokers and nonsmokers was not our comparison of interest but rather served to identify regions exhibiting smoking cue-related activity (the difference between the neural response to smoking vs control scenes) to 
further interrogate these regions for an effect of group. To investigate between-group differences, a region of interest (ROI) analysis was conducted by extracting parameter estimates $(\beta)$ from the contrast of smoking versus control scenes using $6 \mathrm{~mm}$ spherical ROIs centered on the peak voxel of clusters demonstrating an effect of smoking versus control scenes across both groups. In this way, ROIs are defined in an unbiased manner, since both smokers and nonsmokers contribute equally to the statistical parametric map used for ROI selection. Comparisons in a priori regions of the $\mathrm{AON}$ and in cue-reactivity regions are presented at $\alpha=0.05$. Nonhypothesized areas were Bonferroni adjusted for the number of ROIs interrogated $(\alpha=0.003)$.

\section{Results}

Whole-brain random-effects analysis averaging across both smokers and nonsmokers showed greater smoking cuerelated activity in the aIPS, left IFG (BA 45/46), and premotor cortex (Fig. $2 A$ ). In addition, regions commonly implicated in the reward and craving component of drug cue-related activity were also recruited during smoking compared with nonsmoking scenes, namely the dorsal anterior cingulate cortex, orbitofrontal cortex, and dorsolateral prefrontal cortex (Table 1).
A Left Hemisphere

Medial Surface

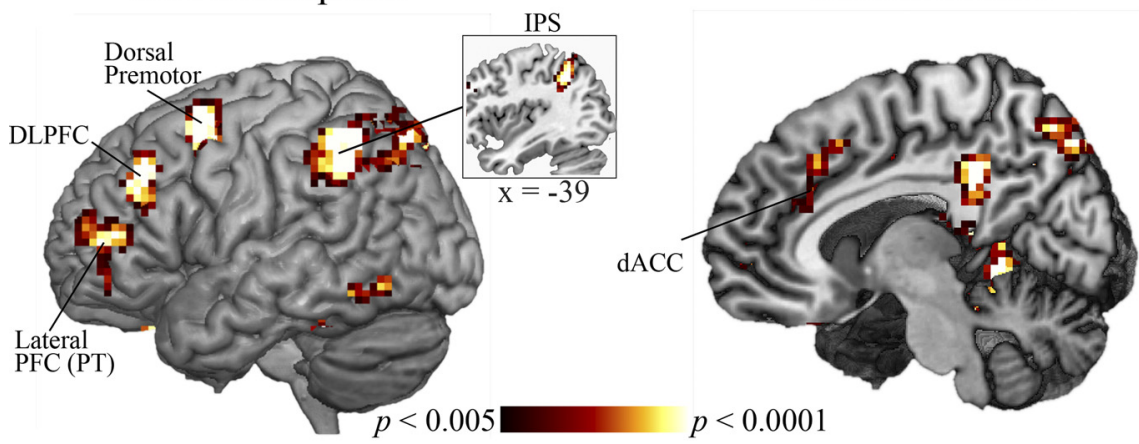

B Left IPS $(-39,-48,42)$
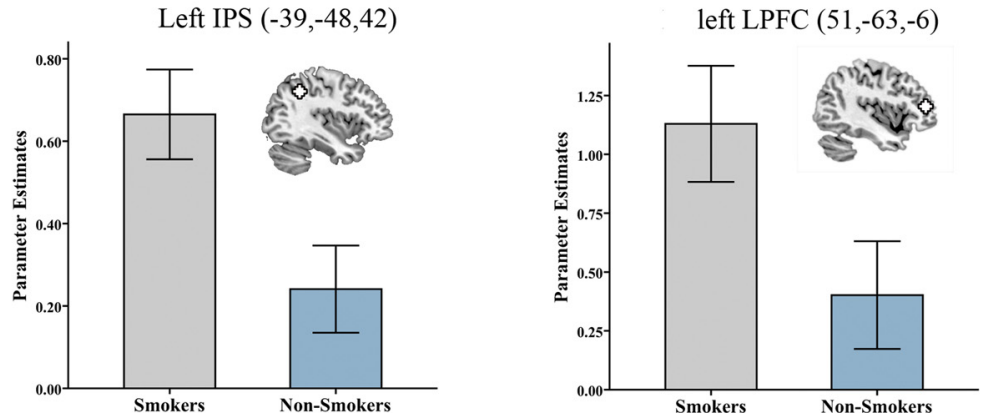

Figure 2. A, Brain regions showing a greater blood oxygenation level-dependent signal response during smoking scenes compared with control scenes in all subjects ( $p<0.005$, uncorrected, cluster extent threshold of 10 contiguous voxels). This parametric map was used to generate unbiased regions of interest to interrogate for an effect of smoking status (i.e., smokers vs nonsmokers). $\boldsymbol{B}$, The left aIPS and IFG demonstrated greater smoking cue-related activity in smokers compared with nonsmokers. Error bars indicate SEM. IPS, Intraparietal sulcus; LPFC, lateral prefrontal cortex; PFC, prefrontal cortex, PT, pars triangularis.
Regions differentiating between smokers and nonsmokers

Regions of interest analysis on ROIs derived from the orthogonal contrast of smoking versus control scenes across all participants revealed between-group differences in both regions of the AON and regions involved in reward and craving. Specifically, smokers demonstrated greater smoking cue-related activity in the left, but not right, aIPS $\left(t_{(32)}=2.8, p=0.009\right)$ and in the left IFG pars triangularis region $\left(t_{(32)}=2.16, p=0.38\right)$ (Fig. $2 B$ ). In addition, smokers demonstrated greater smoking cue-related activity in the dorsal anterior cingulate $\left(t_{(32)}=2.55, p=0.016\right)$, orbiofrontal cortex $\left(t_{(32)}=2.95\right.$, $p=0.006$ ), and bilaterally in the dorsal lateral prefrontal cortex (left: $t_{(32)}=2.61, p=0.014$; right: $\left.t_{(32)}=2.41, p=0.022\right)$. Conversely, nonsmokers did not show greater smoking cue-related activity when compared with smokers in any of the regions identified in the acrosssubject contrast of smoking versus control scenes.

Finally, group differences were specific to the AON and cue reactivity regions. There were no between-group differences in nonhypothesized ROIs from the contrast of smoking versus nonsmoking scenes (both at the corrected $\alpha$ of 0.003 but also uncorrected).

\section{Correlations with postscan cigarette craving}

To examine the relationship between cigarette craving and smoking cue-related activity in smokers, we correlated postscan ratings of cigarette craving (smokers only) with parameter estimates extracted from the ROIs defined above. Only the dorsal anterior cingulate cortex (dACC; MNI coordinates: $9,30,27)$ was significantly correlated with postscan ratings $(r=0.57, p<0.018)$ (Fig. 3).

\section{Discussion}

In this study, we investigated both action representation and reward-related responses to dynamic smoking cues under natural
Table 1. Brain regions showing greater blood oxygenation level-dependent signal response to smoking scenes compared to nonsmoking scenes for all participants (smokers and nonsmokers)

\begin{tabular}{|c|c|c|c|c|c|c|}
\hline \multirow[b]{2}{*}{ Brain region } & \multirow[b]{2}{*}{ Side } & \multirow[b]{2}{*}{ BA } & \multirow[b]{2}{*}{$t$ Value } & \multicolumn{3}{|c|}{$\begin{array}{l}\text { Coordinates of peak } \\
\text { activation }\end{array}$} \\
\hline & & & & $x$ & $y$ & $z$ \\
\hline Anterior intraparietal sulcus & $\mathrm{L}$ & 40 & 5.46 & -39 & -48 & 42 \\
\hline Anterior intraparietal sulcus & $\mathrm{R}$ & 40 & 5.30 & 45 & -48 & 48 \\
\hline Dorsal premotor cortex & $\mathrm{L}$ & 6 & 5.04 & -21 & 9 & 51 \\
\hline Dorsal premotor cortex & $\mathrm{R}$ & 6 & 6.23 & 24 & 12 & 60 \\
\hline Dorsal anterior cingulate cortex & $\mathrm{L}$ & $6 / 32$ & 5.78 & -6 & 24 & 39 \\
\hline Dorsal anterior cingulate cortex & $\mathrm{R}$ & 18 & 3.17 & 9 & 30 & 27 \\
\hline Orbitofrontal cortex & $\mathrm{L}$ & 10 & 3.44 & -33 & 48 & -6 \\
\hline Dorsolateral prefrontal cortex & $\mathrm{L}$ & 9 & 6.32 & -30 & 33 & 36 \\
\hline Dorsolateral prefrontal cortex & $\mathrm{R}$ & 9 & 5.22 & 42 & 30 & 33 \\
\hline Dorsolateral prefrontal cortex & $\mathrm{L}$ & 46 & 4.26 & -42 & 36 & 30 \\
\hline Inferior frontal gyrus & $\mathrm{L}$ & $45 / 46$ & 4.57 & -42 & 45 & 12 \\
\hline Medial fusiform gyrus & $\mathrm{L}$ & - & 19.8 & 21 & -6 & -21 \\
\hline Medial fusiform gyrus & $\mathrm{R}$ & 38 & 10.1 & 42 & 18 & -39 \\
\hline Precuneus & $\mathrm{R}$ & 31 & 4.95 & 9 & -36 & 33 \\
\hline Superior parietal lobule & $\mathrm{L}$ & 7 & 4.32 & -12 & -69 & 48 \\
\hline Superior parietal lobule & $\mathrm{R}$ & 7 & 4.58 & 9 & -78 & 48 \\
\hline Inferior occipital gyrus & $\mathrm{L}$ & 19 & 3.89 & -51 & -63 & -9 \\
\hline
\end{tabular}

Regions showing greater blood oxygenation level-dependent signal response to smoking versus nonsmoking scenes ( $p<0.005$, uncorrected; 10 contiguous voxels) are listed along with the best estimate of their location. Coordinates are in Montreal Neurological Institute (MNI) stereotaxic space.

$B A$, Approximate Brodmann's area; L, left; $R$, right.

viewing conditions. Compared with nonsmokers, smokers showed robust neural activity in response to smoking cues in action-representation regions (left aIPS and left IFG) as well as in cue-reactivity regions [(dACC, orbitofrontal cortex (OFC), and dorsolateral prefrontal cortex (DLPFC)]. The dACC, OFC, and DLPFC are frequently observed in drug cue reactivity studies 


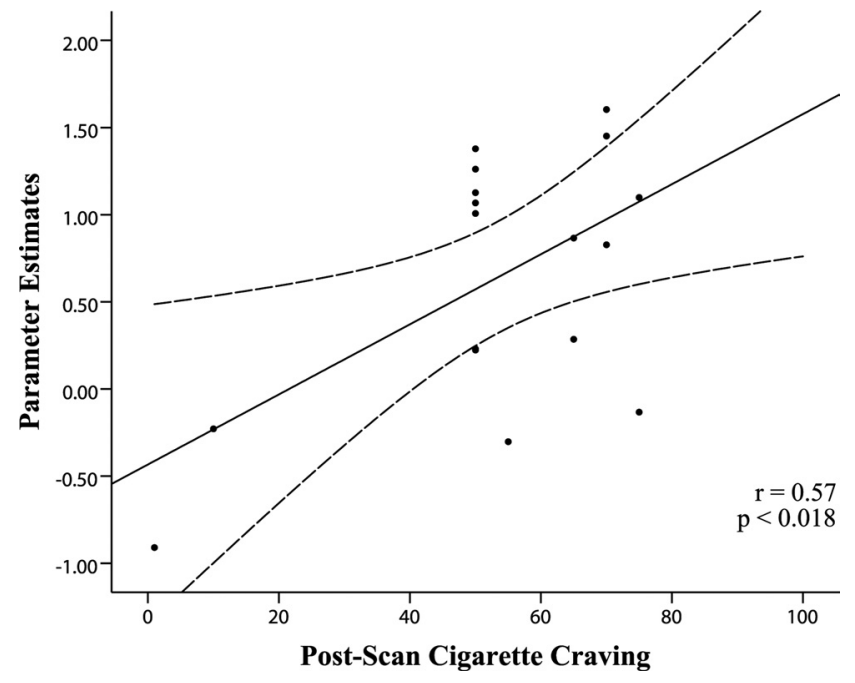

Figure 3. Relationship between postscan cigarette craving in smokers and blood oxygenation level-dependent response difference between smoking and control scenes in the dorsal anterior cingulate cortex $\left(r^{2}=0.33, p<0.018\right.$; MNI coordinates: $\left.9,30,27\right)$. Dashed lines indicate the $95 \%$ confidence intervals.

(Grant et al., 1996; Brody et al., 2002; Due et al., 2002; Wilson et al., 2004; David et al., 2005; McBride et al., 2006) and are thought to be involved in the motivational and cognitive control aspects of drug-seeking behavior (Goldstein and Volkow, 2002). The dACC in particular has often been linked to the subjective experience of craving, and a number of previous studies have demonstrated a correlation between cue reactivity in the dACC and tobacco craving (Brody et al., 2004; Zubieta et al., 2005; McClernon et al., 2005). Here, we replicated the relation between postscan tobacco craving and cue-induced changes in AACC activity, but in this instance it occurred spontaneously in subjects who were unaware that they were being exposed to smoking cues.

Of greater interest was the finding that the left aIPS and left IFG showed greater cue-related activity for smokers than nonsmokers. Both are regions involved in representing goal-directed manual actions, with the left IFG being especially important for planning manual actions (Johnson-Frey et al., 2005). Recently, it has been argued that the aIPS is preferentially tuned to the actions of the contralateral hand. For instance, the left aIPS shows greater activity when viewing right-handed gestures, both when viewing them from the perspective of the person performing the action and when viewing them as though facing the person performing the action (i.e., an allocentric perspective) (Shmuelof and Zohan, 2008). Further evidence comes from a study showing that disrupting activity in the aIPS with TMS impairs grasping of the contralateral, but not the ipsilateral, hand (Rice et al., 2007). In our study, all participants were right-handed, as was the hand used for smoking by the movie's protagonists. This suggests that our finding of differential left, but not right, aIPS activity between smokers and nonsmokers is due in part to this region's bias toward representing actions performed by the contralateral hand, even though, as in Shmuelof and Zohary (2008), all scenes of smoking were observed from an allocentric perspective.

Despite smoking being an inherently manual action, there has been little prior evidence to indicate that when smokers see smoking cues they activate manual action representations in the AON. We propose that this may be largely due to the use of static photographs depicting smoking and smoking paraphernalia by most, but by no means all (Brody et al., 2002), studies of cue reactivity. This is in contradistinction to research on action observation of manual gestures, which relies on dynamic stimuli in the form of video clips (Buccino et al., 2004; Cross et al., 2006; Hamilton and Grafton, 2006; Shmuelof and Zohary, 2008). This is not to say that dynamic stimuli are more ideally suited to drug cue-reactivity research-indeed, static cues have proven instrumental to the study of cue-induced craving — but rather that dynamic action stimuli may be better suited for studying the motor component of drug-taking behavior.

An unanswered question in cue-reactivity research is whether smokers would spontaneously demonstrate neural cue reactivity when smoking cues are processed incidentally. Here, we used a cover story to ensure that smokers remained unaware of the nature of the experiment. In so doing, we avoid potential demand characteristics that can arise when smokers participate in a study in which they either know or can easily infer that they are expected to react to smoking cues. Our findings largely replicate prior neuroimaging research on smoking cue reactivity, demonstrating that the explicit awareness of smoking cues is not essential for the demonstration of drug cue reactivity. In addition, we demonstrated that the previously reported relationship between subjective craving and cue-related activity in the dACC exists even when participants are unaware of the smoking cues.

For smokers trying to quit, scenes of smoking in movies can be a particularly difficult cue to avoid, one that smokers may be unlikely to consider. Research has shown that smoking in movies predicts smoking initiation among adolescents (Dalton et al., 2003, 2009; Hanewinkel and Sargent, 2008) and increases cigarette craving and consumption among adults leaving the movie theater (Sargent et al., 2009; Shmueli et al., 2010). In this study, we demonstrated that smoking in movies, even when processed incidentally, is processed much like an explicit smoking cue and recruits the same brain regions commonly observed in cuereactivity studies. In addition, we demonstrate that watching smoking in movies activates the AON. This network is involved in observation, planning, and motor simulation; it is often characterized as a motor resonance system and forms part of what some researchers have termed the human mirror system (Rizzolatti and Craighero, 2004).

Research on behavioral mimicry has demonstrated that participants spontaneously mimic the incidental actions of others (Chartrand and Bargh, 1999) Recently, this research has been extended to eating behavior, showing that people spontaneously mimic the eating behavior of a confederate, and do so without any awareness of this external influence over their behavior (Johnston, 2002; Tanner et al., 2008). In the present study, smokers demonstrated increased cue-related activity in regions of the AON, suggesting that they were spontaneously representing the action of smoking. Due the physical constraints of the scanner environment, we were not able to assess behavioral mimicry, but the prior research outlined above suggests seeing smoking in a movie would increase the likelihood that a smoker would subsequently light up a cigarette.

Studies of drug cue reactivity have largely focused on the link between cue-induced craving and drug use; however, it has long been suggested that another route through which drug cues may induce increased drug use is through activation of conditioned sensorimotor associations (Tiffany, 1990). Further study of this automatic component of cue reactivity, especially in middle-aged smokers who have decades of smoking experience, may help elucidate why smokers trying to quit find it more difficult to overcome the habitual and automatic aspects of smoking compared with physical withdrawal (Russell et al., 1974; Berlin et al., 2003). 
Moreover, for smokers attempting abstinence, scenes of smoking in movies is a particularly difficult cue to escape, given the ubiquity of movies and television. Although an abstinent smoker can remove smoking paraphernalia from the home and avoid highrisk situations that may lead to smoking relapse, they are unlikely to refrain from watching a movie due to its smoking content. Finally, our results further the understanding of how complex human behaviors can be shaped by subtle contextual cues, such as how smokers might be tempted to share a cigarette with onscreen smokers.

\section{References}

Bargh JA, Ferguson MJ (2000) Beyond behaviorism: on the automaticity of higher mental processes. Psychol Bull 126:925-945.

Berlin I, Singleton EG, Pedarriosse AM, Lancrenon S, Rames A, Aubin HJ, Niaura R (2003) The modified reasons for smoking scale: factorial structure, gender effects and relationship with nicotine dependence and smoking cessation in French smokers. Addiction 98:1575-1583.

Brody AL, Mandelkern MA, London ED, Childress AR, Lee GS, Bota RG, Ho ML, Saxena S, Baxter LR Jr, Madsen D, Jarvik ME (2002) Brain metabolic changes during cigarette craving. Arch Gen Psychiatry 59:11621172.

Brody AL, Mandelkern MA, Lee G, Smith E, Sadeghi M, Saxena S, Jarvik ME, London ED (2004) Attenuation of cue-induced cigarette craving and anterior cingulate cortex activation in bupropion-treated smokers: a preliminary study. Psychiatry Res 130:269-281.

Buccino G, Vogt S, Ritzl A, Fink GR, Zilles K, Freund HJ, Rizzolatti G (2004) Neural circuits underlying imitation learning of hand actions: an event related fMRI study. Neuron 42:323-334.

Calvo-Merino B, Glaser DE, Grèzes J, Passingham RE, Haggard P (2005) Action observation and acquired motor skills: an fMRI study with expert dancers. Cereb Cortex 15:1243-1249.

Chartrand TL, Bargh JA (1999) The chameleon effect: the perceptionbehavior link and social interaction. J Pers Soc Psychol 76:893-910.

Cross ES, Hamilton AF, Grafton ST (2006) Building a motor simulation de novo: Observation of dance by dancers. Neuroimage 31:1257-1267.

Dalton MA, Sargent JD, Beach ML, Titus-Ernstoff L, Gibson JJ, Ahrens MB, Tickle JJ, Heatherton TF (2003) Effect of viewing smoking in movies on adolescent smoking initiation: a cohort study. Lancet 362:281-285.

Dalton MA, Beach ML, Adachi-Mejia AM, Longacre MR, Matzkin AL, Sargent JD, Heatherton TF, Titus-Ernstoff L (2009) Early exposure to movie smoking predicts established smoking by older teens and young adults. Pediatrics 123:e551-e558.

David SP, Munafò MR, Johansen-Berg H, Smith SM, Rogers RD, Matthews PM, Walton RT (2005) Ventral striatum/nucleus accumbens activation to smoking-related pictorial cues in smokers and nonsmokers: a functional magnetic resonance imaging study. Biol Psychiatry 58:488-494.

Decety J, Perani D, Jeannerod M, Bettinardi V, Tadary B, Woods R, Mazziotta JC, Fazio F (1994) Mapping motor representations with positron emission tomography. Nature 371:600-602.

Due DL, Huettel SA, Hall WG, Rubin DC (2002) Activation in mesolimbic and visuospatial neural circuits elicited by smoking cues: evidence from functional magnetic resonance imaging. Am J Psychiatry 159:954-960.

Field M, Mogg K, Bradley BP (2006) Automaticity of smoking behaviour: the relationship between dual-task performance, daily cigarette intake and subjective nicotine effects. J Psychopharmacol 20:799-805.

Goldstein RZ, Volkow ND (2002) Drug addiction and its underlying neurobiological basis: neuroimaging evidence for the involvement of the frontal cortex. Am J Psychiatry 159:1642-1652.

Grafton ST, Arbib MA, Fadiga L, Rizzolatti G (1996) Localization of grasp representations in humans by positron emisson tomography. 2. Observation compared with imagination. Exp Brain Res 112:103-111.

Grant S, London ED, Newlin DB, Villemagne VL, Liu X, Contoreggi C, Phillips RL, Kimes AS, Margolin A (1996) Activation of memory circuits during cue-elicited cocaine craving. Proc Natl Acad Sci U S A 93: $12040-12045$.

Hamilton AF, Grafton ST (2006) Goal representation in human anterior intraparietal sulcus. J Neurosci 26:1133-1137.

Hanewinkel R, Sargent JD (2008) Exposure to smoking in internationally distributed American movies and youth smoking in Germany: a crosscultural cohort study. Pediatrics 121:e108-e117.

James W (1890) Principles of psychology, Vol 1. New York: Cosimo.

Johnson-Frey SH, Maloof FR, Newman-Norlund R, Farrer C, Inati S, Grafton ST (2003) Actions or hand-object interactions? Human inferior frontal cortex and action observation. Neuron 39:1053-1058.

Johnson-Frey SH, Newman-Norlund R, Grafton ST (2005) A distributed left hemisphere network active during planning of everyday tool use skills. Cereb Cortex 15:681-695.

Johnston L (2002) Behavioral mimicry and stigmatization. Soc Cogn 20:18-35.

McBride D, Barrett SP, Kelly JT, Aw A, Dagher A (2006) Effects of expectancy and abstinence on the neural response to smoking cues in cigarette smokers: an fMRI study. Neuropsychopharmacology 31:2728-2738.

McClernon FJ, Hiott FB, Huettel SA, Rose JE (2005) Abstinence-induced changes in self-report craving correlate with event-related fMRI responses to smoking cues. Neuropsychopharmacology 30:1940-1947.

Moll J, de Oliveira-Souza R, Passman LJ, Cunha FC, Souza-Lima F, Andreiuolo PA (2000) Functional MRI correlates of real and imagined tool-use pantomimes. Neurology 54:1331-1336.

Moran JM, Wig GS, Adams RB Jr, Janata P, Kelley WM (2004) Neural correlates of humor detection and appreciation. Neuroimage 21:1055-1060.

Rice NJ, Tunik E, Cross ES, Grafton ST (2007) On-line grasp control is mediated by the contralateral hemisphere. Brain Res 1175:76-84.

Rizzolatti G, Craighero L (2004) The mirror-neuron system. Annu Rev Neurosci 27:169-192.

Rizzolatti G, Fadiga L, Matelli M, Bettinardi V, Paulesu E, Perani D, Fazio F (1996) Localization of grasp representations in humans by PET. 1. Observation versus execution. Exp Brain Res 111:246-252.

Russell M, Peto J, Patel U (1974) The classification of smoking by factorial structure of motives. J R Stat Soc 137:313-346.

Sargent JD, Worth KA, Beach M, Gerrard M, Heatherton TF (2008) Population-based assessment of exposure to risk behaviors in motion pictures. Commun Methods Meas 2:134-151.

Sargent JD, Morgenstern M, Isensee B, Hanewinkel R (2009) Movie smoking and urge to smoke among adult smokers. Nicotine Tob Res 11: 1042-1046.

Shmueli D, Prochaska JJ, Glantz SA (2010) Effect of smoking scenes in films on immediate smoking: a randomized controlled study. Am J Prev Med 38:351-358.

Schmuelof L, Zohary E (2005) Dissociation between ventral and dorsal fMRI activation during object and action recognition. Neuron 47:457-470.

Shmuelof L, Zohary E (2008) Mirror-image representation of action in the anterior parietal cortex. Nat Neurosci 11:1267-1269.

Tanner RJ, Ferraro R, Chartrand TL, Bettman JR, Van Baaren R (2008) Of chameleons and consumption: the impact of mimicry on choice and preferences. J Consum Res 34:754-766.

Tiffany ST (1990) A cognitive model of drug urges and drug-use behavior: role of automatic and nonautomatic processes. Psychol Rev 97:147-168.

Wilson SJ, Sayette MA, Fiez JA (2004) Prefrontal responses to drug cues: a neurocognitive analysis. Nat Neurosci 7:211-214.

Zubieta J, Heitzeg MM, Xu Y, Koeppe RA, Ni L, Guthrie S, Domino EF (2005) Regional cerebral blood flow responses to smoking in tobacco smokers after overnight abstinence. Am J Psychiatry 162:567-577. 